\title{
Ratnagiri 7 - Red Kernel, Semi Dwarf and High Yielding Rice Variety for Konkan Region of Maharashtra State in India
}

\author{
B.D. Waghmode ${ }^{1 *}$, N.G. Sonone ${ }^{1}$, V.C. Navhale ${ }^{1}$, S.R. Kadam ${ }^{1}$ and S.G. Bhave ${ }^{2}$ \\ ${ }^{1}$ Agricultural Research Station, Shirgaon, Dist. Ratnagiri - 415629 (MS), India \\ ${ }^{2}$ Director of Extension, Dr Balasaheb Sawant Konkan Krishi Vidyapeeth, \\ Dapoli-415 712, India \\ *Corresponding author
}

\begin{abstract}
A B S T R A C T
The rice variety Ratnagiri 7 (Red rice; RTNRR-4) was evolved through the mutant selection from MO17. The above variety is midlate in duration (122-125 days), Semidwarf (100-110 cm plant height), short bold grain type. The variety showed 46.50, 21.40, 50.96 and 59.41 per cent higher yield over the respective checks in station, state, agronomical and adaptive trials, respectively. Ratnagiri 7 (RTN RR-4) recorded 23.88\%, $18.69 \%$ and $39.40 \%$ higher grain yield over IR-64, BPT-5204 and Kalanamak respectively in AICRIP trials. RTN RR-4 consists of 28.50\% (17.35 ppm) and 32.7\% (7.9 ppm) high iron content over the check Bela (13.5 and $5.95 \mathrm{ppm})$ in brown and polished rice respectively. Ratnagiri 7 (RTN RR-4) consists of $42.82 \%$ (28.35 ppm) and $43.0 \%$ (24.25 ppm) high zinc content over the check Bela (19.85 and $16.95 \mathrm{ppm})$ in brown and polished rice respectively. It also having low glycemic index (53). RTNRR-4 having high milling $(64.17 \%)$ and head rice recovery percentage $(60.65 \%)$ with good cooking quality. It recorded average yield of 4.5 to 5.0 t/ha. It showed resistant reaction to stem borer, leaf folder and gall midge and moderately resistant to leaf blast, bacterial leaf blight. Therefore the rice variety Ratnagiri 7 recommended for release for commercial cultivation in Konkan region of Maharashtra in the year 2017.
\end{abstract}

\section{Keywords}

Ratnagiri 7, Zinc, Iron, Red rice, Medium, Grain yield

Article Info

Accepted:

28 March 2018

Available Online:

10 April 2018

\section{Introduction}

Rice is a staple food for above 60 per cent world's population. It is wholesome and nutritious cereal and source of complete carbohydrate. The world's capacity to sustain a favourable food production/population balance has again come under the spotlight in view of continued population increase and a drastic slowdown in growth of cereal production. According to United Nations estimates, the world population will grow to 8 billion in 2025 requiring about $40 \%$ more rice production to cater demand of the burgeoning global population. India is the foremost country of the world in area of rice cultivation and second to China in rice production. The total area under rice cultivation in India is about 44.11 million ha with a rough rice production of 105.48 million tons (Anonymous, 2017).

In Maharashtra, rice is the second most important food grain crop of the people, which 
grown over an area of 15.57 lakh hectares with an annual rough rice and milled rice production of 52.95 lakh tones and 26.54 lakh tones, respectively. The average productivity rough rice and rice of the state is $3.4 \mathrm{t} / \mathrm{ha}$ and $2.35 \mathrm{t} / \mathrm{ha}$. The average productivity of the state is stable, which is low as compared to other rice producing states like Punjab (3.84 t/ha), Tamil Nadu (3.19 t/ha), Telangana (3.14 t/ha), Haryana (3.11 t/ha), Andhra Pradesh (3.02t/ha), West Bengal (2.73 t/ha), Karnataka (2.67 t/ha), Gujarat (2.33 t/ha) and national productivity (2.39 t/ha) (Anonymous, 2017).

Konkan region is a major rice producing area of Maharashtra. Nearly 3.69 lakh ha area of Konkan is under rice crop with rough rice production of 15.70 lakh tones. The average rough rice productivity of the Konkan region is 4.25 t/ha. Konkan region contributes $23.70 \%$ in area under rice crop and produces $29.65 \%$ rough rice at state level (Anonymous, 2017).

In some areas of India, red rice are considered highly nutritive and medicinal. The rice is eaten as whole grain; Red gunja is preferred for making bread and chapati (Rani and Krishnaiah, 2001). Glutinous rice is used in making puttu in South India. In Himachal Pradesh, Jatu red rice is prized for its aroma and taste. Matali and Laldhan of Himachal Pradesh are used for curing blood pressure and fever. Kafalya, from the hills of Himachal Pradesh and Uttar Pradesh, is used for treating leucorrhea and abortion complications. Karikagga and Atikaya of Karnataka are used for coolness and as tonic, while Neelamsamba is used for lactating mothers in Tamil Nadu (Arumugasamy et al., 2001).

In addition of being nutritive and having medicinal value, red rice possesses many other special features. It is common experience that red and black husked rice is comparatively more resistant to storage insect pests than brown husked rice. The Patni rice of Maharashtra and the Jatu of Himachal Pradesh are well known for such hardiness and resistance. In addition to storage capability, red rice varieties suitable for various agroclimatic conditions and adverse situations are also commonly cultivated in certain regions. Agronomically, or from the cultivation point of view, such rices possess resistance to drought, flood, submergence, alkalinity, salinity, and resistance to pests and diseases (Chaudhary and Tran, 2001).

In Maharashtra, particularly in Sindhudurg, Ratnagiri, Raigad and Thane districts, local red kernel types are grown since ancient years. These varieties are Patni, Munga, Bela, Valai, Halga Red, Kala Rata, Bura Rata, Jaddu, Varangal etc. These local red rices are mainly having bold grain type, tall and grassy stature, seed dormancy, sparse plant type, lodging and low yields. However, these varieties are having low amylose, high zinc, iron, riboflavin and antioxidant properties. Their nutritional and physico-chemical properties are used for making soup; locally known as Pej and served to children, women and patients for their daily breakfast. Considering these attributes, red rice could once again find favour with health conscious consumers. It is high time that people in India took a fresh look at similar properties in the vast pool of indigenous red rice. So there is a need for revival of red rice in India and new cultivars should be released. There is also a dire need for clinical validation of the medicinal value of red rice reported in ancient literature, and for research on food preparations from red rice. Hence, the efforts being made to develop $\mathrm{Zn}$ and Iron rich rice with modern plant stature. Ratnagiri 7 (RTN RR-4) is semi dwarf $(100-110 \mathrm{~cm})$, midlate in duration (122-125 days), high Iron and Zinc content, resistant to stem borer, leaf folder, gall midge and moderately resistant to leaf blast and bacterial leaf blight. Short bold grain, non-lodging, 
non-shattering with average yield 4.5 to 5.0 t/ha. Therefore, the release proposal of RTNRR-4 rice variety is submitted for recommendation in the Konkan, Western Maharashtra and Vidarbha region.

\section{Materials and Methods}

RTNRR-4 is a mutant selection from MO-17. The selections were made for high zinc and iron content with high grain yield. Among the several selections in mutant populations, a fixed mutant line RTNRR-4 was selected from MO17. The fixed line, RTNRR4 was further tested in various trials viz., station trials, state coordinated trials and national coordinated trials at various locations in the state and country. This culture also tested in agronomical trial at Agricultural Research Station, Shirgaon, Ratnagiri, MS during Kharif 2016. The 15 adoptive trials were conducted during Kharif 2016 in five districts of Konkan region of Maharashtra state. The culture was screened for resistance to various insect pests and diseases at endemic sites. The physical and biochemical quality parameters were analyzed at Regional Agricultural Research Station, Karjat. Zinc and iron analysis was done ICAR- IIRR., Rajendranagar, Hyderabad -30 and Hi Tech. Lab., Sangli. The yield data of various trials were statistically analysed according to Panse and Sukhatme (1967). Based on yield data of various trials, superior grain quality, resistance for disease and insect pest reactions and consistent yield performance at various test locations, Ratnagiri 7 (RTN RR-4) rice variety was recommended to release for Konkan region of state of Maharashtra for commercial cultivation during the year 2017.

\section{Results and Discussion}

The yield performance of Ratnagiri 7 (Red rice) (IET-25448) rice variety in various trials conducted during 2011 to 2016 , is presented in Table 1. In station trials conducted during
Kharif 2012, 2013, 2014 and 2015, RTN RR-4 has recorded $66.34 \%, 15.86 \%, 54.43 \%$ and $42.87 \%$ higher grain yield over the check Bela respectively. Ratnagiri 7 (RTNRR-4) rice variety recorded $66.34 \%$ higher grain yield (6414 kg/ha) over check Bela (3856) kg/ha in Initial Varietal Trial (Station) conducted during Kharif 2012, similarly, during Kharif 2013 in IVT (Station) it has recorded 3506 $\mathrm{kg} / \mathrm{ha}$ of grain yield which was $15.86 \%$ higher than the check Bela. Ratnagiri 7 (RTNRR-4) rice variety recorded $54.43 \%$ higher grain yield (5660 kg/ha) over check Bela (3665) $\mathrm{kg} / \mathrm{ha}$ in Advance Varietal Trial (Station) conducted during Kharif 2014, similarly, during Kharif 2015 in AVT (Station) it has recorded $5072 \mathrm{~kg} / \mathrm{ha}$ of grain yield which was $42.87 \%$ higher than the check Bela. On the basis of overall four years performance of RTN RR-4, it was found $46.50 \%$ higher in grain yield $(5163 \mathrm{~kg} / \mathrm{ha})$ against the check Bela (3524 kg/ha) (Table 1) (Anonymous, 2017).

Ratnagiri 7 (RTN RR-4) has recorded $11.02 \%$ (4504 kg/ha), $23.36 \%$ (4227 kg/ha) and $28.2 \%$ $(5536 \mathrm{~kg} / \mathrm{ha})$ higher grain yield over the check Bela (4057 kg/ha, $3427 \mathrm{~kg} / \mathrm{ha} \& 4318 \mathrm{~kg} / \mathrm{ha})$ in Maharashtra state coordinated trials viz., Initial varietal Trial, Advance Varietal Trial-I and Advance Varietal Trial-II conducted during Kharif 2013, Kharif 2014, and Kharif 2016 respectively (Table 1 ). On the basis of overall three years performance of RTN RR-4 in state coordinated trials conducted at nine locations in Maharashtra state, it was found superior by $21.40 \%(4744 \mathrm{~kg} / \mathrm{ha})$ than the check Bela (3908 kg/ha) (Table 1).

Ratnagiri 7 was evaluated in IVT-(MS) All India Coordinated Trials under as RTN RR-4 (IET-25448) testing code at 22 locations during Kharif- 2015 in the country. It recorded $23.88 \%$ higher grain yield over national check IR-64 (Medium) (3593 kg/ha), 18.69\% higher grain yield over BPT-5204 (Late) (3750 $\mathrm{kg} / \mathrm{ha}$ ) and $39.40 \%$ higher grain yield over 
Kalanamak (Quality) (3193 kg/ha). In AICRIP Trial IVT-(M) conducted during Kharif-2015 season indicating it's wider adaptability in varied agro-ecological situations in the country (Anonymous, 2017).

The rice culture, Ratnagiri 7 (RTNRR-4) (4890 kg/ha) has recorded $59.41 \%$ increase in grain yield over check Bela (3067 kg/ha) in 15 adaptive trials conducted during Kharif 2016 on farmers' fields (Table 2).

The experiment was conducted at ARS, Shirgaon (Ratnagiri) to study the effect of spacing and nitrogen levels along with common dose of phosphorus and potash on red rice genotype RTN R-R 4. The response of red rice genotype RTN R-R 4 had significant effect on grain yield. The red rice genotype RTN R-R 4 produced higher varietal response (41.20 Q/ha) over check i.e. Bela cultivar $(23.79 \mathrm{Q} / \mathrm{ha})$ and its increment was to the tune of $42.26 \%$. The response of red rice genotype RTN R-R 4 to nitrogen levels was statistically significant. The level of nitrogen $120 \mathrm{~kg} \mathrm{~N} / \mathrm{ha}$ (35.39 Q/ha) and $150 \mathrm{~kg} \mathrm{~N} / \mathrm{ha}(32.55 \mathrm{Q} / \mathrm{ha})$ produced significantly higher grain yield over $100 \mathrm{~kg} \mathrm{~N} / \mathrm{ha}$. Red rice genotype RTN R-R 4 grown at closer spacing of $15 \times 15 \mathrm{~cm}$ recorded significantly higher grain yield ( $35.54 \mathrm{Q} / \mathrm{ha}$ ) over $20 \times 15 \mathrm{~cm}$ and $20 \times 20 \mathrm{~cm}$ spacing (Table 3).

Individual interactions between genotypes/variety x spacing, genotypes/variety $\mathrm{x}$ nitrogen levels and spacing $\mathrm{x}$ nitrogen levels were found to be statistically significant. Red rice genotype RTN R-R 4 grown at closer spacing $15 \times 15 \mathrm{~cm}$ produced significantly higher grain yield $(46.54 \mathrm{Q} / \mathrm{ha})$ over rest of combinations and that increment was to the tune of $47.25 \%$ when rice cultivar Bela was grown at same spacing. Red rice genotype RTN R-R 4 fertilized with $120 \mathrm{~kg} \mathrm{~N} / \mathrm{ha}$ noticed significantly highest grain yield (45.51
Q/ha) and it was to the magnitude of $44.48 \%$, when rice cultivar Bela was grown with same nitrogen level. Moreover, 15 x $15 \mathrm{~cm}$ plant spacing and $120 \mathrm{~kg} \quad \mathrm{~N} / \mathrm{ha}$ produced significantly higher grain yield $(40.15 \mathrm{Q} / \mathrm{ha})$ over all other treatment combination (Table $3)$.

From this results of this experiment, it is concluded that, the rice genotype, RTN R-R 4 produced higher grain yield $53.87 \mathrm{q} / \mathrm{ha}$ by adopting $15 \times 15 \mathrm{~cm}$ spacing and $120 \mathrm{~kg} \mathrm{~N}+$ $50 \mathrm{~kg} \mathrm{P}_{2} \mathrm{O}_{5}+50 \mathrm{~kg} \mathrm{~K}_{2} \mathrm{O} / \mathrm{ha}$ as fertilizer dose, which was $50.96 \%$ higher over cultivar Bela by adopting same package of practice during kharif season (Table 3).

Ratnagiri 7 (Red rice) rice variety is midlate in duration (122-125 days duration in Kharif and 130-135 days duration in rabi/hot weather seasons), Semi dwarf (100-110 cm plant height), Medium slender kernel type (M.S.), average 1000 kernel weight of $25.8 \mathrm{~g}$ with an average grain yield of 4.5 to $5.0 \mathrm{t} / \mathrm{ha}$. The variety is non-lodging and non-shattering type. The details of salient features and characterization as per DUS guideline of Ratnagiri 7 (IET-25448) are presented in Table 4 and 5.

RTN RR-4 is red kernel variety consists of $28.50 \%$ (17.35 ppm) and 32.77\% (7.90 ppm) high iron content over the check Bela (13.50 and $5.95 \mathrm{ppm}$ ) in brown and polished rice respectively in two years testing. It consists of $42.82 \%$ (28.35 ppm) and $43.00 \%$ (24.25 ppm) high zinc content over the check Bela (19.85 and $16.95 \mathrm{ppm}$ ) in brown and polished rice respectively in two years testing. It also content low glycemic Index (53) (Anonymous 2017). The milling and cooking qualities of Ratnagiri 7 (Red rice) rice variety was estimated at Regional Agricultural Research Station, Karjat during 2015. It showed excellent kernel quality features. 
Table.1 Yield performance of Ratnagiri 7 (IET-25448) in different trials and demonstrations

\begin{tabular}{|l|c|c|c|c|c|}
\hline Particulars & Year & \multicolumn{2}{|l|}{$\begin{array}{l}\text { Average grain yield (kg/ha) } \\
\text { Ratnagiri 7 } \\
\text { Check Bela }\end{array}$} & $\begin{array}{l}\text { Name of the } \\
\text { check }\end{array}$ & $\begin{array}{l}\text { Per cent } \\
\text { increase } \\
\text { over check }\end{array}$ \\
\hline Initial Station trial & 2012 & $6414^{* *}$ & 3856 & Bela & 66.34 \\
\hline Initial Station trial & 2013 & $3506^{*}$ & 3026 & Bela & 15.86 \\
\hline $\begin{array}{l}\text { Advance Station trial } \\
\text { Advance Station trial }\end{array}$ & 2014 & $5660^{* *}$ & 3665 & Bela & 54.43 \\
\hline $\begin{array}{l}\text { Initial State Coordinated trial (9 } \\
\text { locations) }\end{array}$ & 2015 & $5072^{* *}$ & 3550 & Bela & 42.87 \\
\hline $\begin{array}{l}\text { Advance State Coordinated trial } \\
\text { (AVT-I) (9 locations) }\end{array}$ & 2014 & $4504^{*}$ & 4057 & Bela & 11.02 \\
\hline $\begin{array}{l}\text { Advance State Coordinated trial } \\
\text { (AVT-II) (9 locations) }\end{array}$ & 2015 & $5227^{*}$ & 3427 & Bela & 23.34 \\
\hline AICRIP trial- SG (22 locations) & 2015 & $4451^{* *}$ & 4318 & Bela & 28.21 \\
\hline $\begin{array}{l}\text { Adaptive trial (15 locations) } \\
\text { Agronomical trial }\end{array}$ & 2016 & $4890^{* *}$ & 3193 & Kalanamak & 39.40 \\
\hline Average & 2016 & $5387^{* *}$ & 2642 & Bela & 103.89 \\
\hline
\end{tabular}

*, ** Significant at $5 \%$ and $1 \%$ respectively

(Anonymous, 2017)

Table.2 Yield performance of Ratnagiri 7 (Red rice) (RTNRR-4) under adaptive trials conducted on farmers' field in five districts of Konkan region during Kharif 2016

\begin{tabular}{|c|c|c|c|c|c|}
\hline \multirow{2}{*}{$\begin{array}{l}\text { Sr. } \\
\text { No. }\end{array}$} & \multirow[t]{2}{*}{ Districts } & \multirow{2}{*}{$\begin{array}{c}\text { No. of } \\
\text { Adaptive trials }\end{array}$} & \multicolumn{2}{|c|}{ Grain Yield (kg/ha) } & \multirow{2}{*}{$\begin{array}{l}\% \text { increase } \\
\text { over check }\end{array}$} \\
\hline & & & RTN RR-4 & CH. Bela & \\
\hline 1 & Sindhudurg & 03 & 4850 & 3140 & 54.46 \\
\hline 2 & Ratnagiri & 03 & 5140 & 3380 & 52.07 \\
\hline 3 & Raigad & 03 & 5325 & 3012 & 76.79 \\
\hline 4 & Thane & 03 & 4822 & 2950 & 63.46 \\
\hline 5 & Palghar & 03 & 4312 & 2855 & 51.03 \\
\hline \multicolumn{2}{|c|}{ Total/A verage } & 15 & 4890 & 3067 & 59.41 \\
\hline
\end{tabular}

Table.3 Effect of different levels of nitrogen on grain yield (q/ha) of rice genotype Ratnagiri 7 (Red rice) (RTNRR-4) during Kharif 2016

\begin{tabular}{|c|c|c|c|c|c|}
\hline \multirow[t]{2}{*}{ Genotypes } & \multirow[t]{2}{*}{ Spacing } & \multicolumn{4}{|c|}{ Nitrogen levels } \\
\hline & & $\begin{array}{c}\mathbf{N}_{\mathbf{1}} \\
(100 \mathrm{~kg} \mathrm{~N} / \mathrm{ha})\end{array}$ & $\begin{array}{c}\mathbf{N}_{\mathbf{2}} \\
(120 \mathrm{~kg} \mathrm{~N} / \mathrm{ha})\end{array}$ & $\begin{array}{c}\mathbf{N}_{\mathbf{3}} \\
(150 \mathrm{~kg} \\
\mathrm{N} / \mathrm{ha})\end{array}$ & Mean \\
\hline \multirow{3}{*}{$\begin{array}{c}\mathrm{V}_{1} \\
(\mathrm{RTN} \text { R-R 4) }\end{array}$} & $\mathrm{S}_{1}(15 \times 15 \mathrm{~cm})$ & 38.33 & 53.87 & 47.42 & 46.54 \\
\hline & $\mathrm{S}_{2}(20 \times 15 \mathrm{~cm})$ & 35.69 & 44.34 & 39.11 & 39.71 \\
\hline & $\mathrm{S}_{3}(20 \times 20 \mathrm{~cm})$ & 36.05 & 38.32 & 37.65 & 37.34 \\
\hline \multirow{4}{*}{$\begin{array}{c}\mathrm{V}_{2} \\
\text { (Bela) }\end{array}$} & $\mathrm{S}_{1}(15 \times 15 \mathrm{~cm})$ & 22.59 & 26.42 & 24.63 & 24.55 \\
\hline & $\mathrm{S}_{2}(20 \times 15 \mathrm{~cm})$ & 23.24 & 25.52 & 23.49 & 24.08 \\
\hline & $\mathrm{S}_{3}(20 \times 20 \mathrm{~cm})$ & 21.33 & 23.85 & 23.02 & 22.73 \\
\hline & Mean & 29.54 & 35.39 & 32.55 & 32.49 \\
\hline
\end{tabular}




\begin{tabular}{c|c|c|c|c|}
\hline Parameters & Variety & Spacing & Nitrogen levels & $\begin{array}{c}\text { Interaction (Variety X Spacing X } \\
\text { Nitrogen) }\end{array}$ \\
\hline S.E. \pm & 0.917 & 0.764 & 0.655 & 1.605 \\
\hline CD $(\mathbf{0 . 0 5})$ & 5.579 & 2.492 & 1.912 & NS \\
\hline
\end{tabular}

(Anonymous, 2017)

Table.4 Characterization of Ratnagiri 7 (RTNRR-4) as per DUS guidelines

\begin{tabular}{|c|c|c|c|c|c|}
\hline $\begin{array}{l}\text { Sr. } \\
\text { No }\end{array}$ & Characteristics & Particulars & $\begin{array}{l}\text { Sr. } \\
\text { No }\end{array}$ & Characteristics & Particulars \\
\hline 1. & Coleoptile: Colour & Green (2) & 19. & Culm: attitude & Erect (1) \\
\hline 2. & Basal leaf: Sheath colour & Green (1) & 20. & $\begin{array}{l}\text { Time of heading ( } 50 \% \text { of } \\
\text { plants with panicles) }\end{array}$ & Early (3) \\
\hline 3. & $\begin{array}{l}\text { Leaf: Intensity of green } \\
\text { colour }\end{array}$ & Medium (5) & 21. & $\begin{array}{l}\text { Flag leaf: Attitude } \\
\text { of blade (early observation) }\end{array}$ & Erect (1) \\
\hline 4. & $\begin{array}{l}\text { Leaf: Anthocyanin } \\
\text { colouration }\end{array}$ & Absent (1) & 22. & $\begin{array}{l}\text { Spikelet: Density } \\
\text { of pubescence of lemma }\end{array}$ & Weak (3) \\
\hline 5. & $\begin{array}{l}\text { Leaf: Distribution of } \\
\text { anthocyanin colouration }\end{array}$ & NA & 23. & Male sterility & Absent (1) \\
\hline 6. & $\begin{array}{l}\text { Leaf Sheath: anthocyanin } \\
\text { Colouration }\end{array}$ & Absent (1) & 24. & $\begin{array}{l}\text { Lemma: Anthocyanin } \\
\text { colouration of keel }\end{array}$ & Absent (1) \\
\hline 7. & $\begin{array}{l}\text { Leaf sheath: Intensity of } \\
\text { Anthocyanin colouration }\end{array}$ & NA & 25. & $\begin{array}{l}\text { Lemma: Anthocyanin } \\
\text { colouration of area below } \\
\text { apex }\end{array}$ & Absent (1) \\
\hline 8. & $\begin{array}{l}\text { Leaf: Pubescence } \\
\text { of blade surface }\end{array}$ & Weak (3) & 26. & $\begin{array}{l}\text { Lemma: Anthocyanin } \\
\text { colouration of apex }\end{array}$ & Absent (1) \\
\hline 9. & Leaf: Auricles & Present (9) & 27. & Spikelet: Colour of stigma & White (1) \\
\hline 10. & $\begin{array}{l}\text { Leaf: Anthocyanin } \\
\text { colouration of auricles }\end{array}$ & $\begin{array}{l}\text { Colourless } \\
\text { (1) }\end{array}$ & 28. & Stem: Thickness & Medium (5) \\
\hline 11. & Leaf: Collar & Present (9) & 29. & $\begin{array}{l}\text { Stem: Length (excluding } \\
\text { panicle; excluding floating } \\
\text { rice) }\end{array}$ & Very Short (1) \\
\hline 12. & $\begin{array}{l}\text { Leaf: Anthocyanin } \\
\text { colouration of collar }\end{array}$ & Absent (1) & 30. & $\begin{array}{l}\text { Stem: Anthocyanin } \\
\text { colouration of nodes }\end{array}$ & Absent (1) \\
\hline 13. & Leaf: Ligule & Present (9) & 31. & $\begin{array}{l}\text { Stem: Intensity of thiocyanin } \\
\text { coloration of } \\
\text { nodes }\end{array}$ & NA \\
\hline 14. & Leaf: Shape of ligule & Split (3) & 32. & $\begin{array}{l}\text { Stem: Anthocyanin } \\
\text { colouration of internodes }\end{array}$ & Absent (1) \\
\hline 15. & Leaf: Colour of ligule & White (1) & 33. & $\begin{array}{l}\text { Panicle: Length of } \\
\text { main axis }\end{array}$ & $\begin{array}{l}\text { Medium } \\
(21-25 \mathrm{~cm})(5)\end{array}$ \\
\hline 16. & Leaf: Length of blade & Short (3) & 34. & $\begin{array}{l}\text { Flag leaf: Attitude of blade } \\
\text { (late observation) }\end{array}$ & Semi erect (3) \\
\hline 17. & Leaf: Width of blade & Medium (5) & 35. & $\begin{array}{l}\text { Panicle: Curvature of main } \\
\text { axis }\end{array}$ & $\begin{array}{l}\text { Semi straight } \\
\text { (3) }\end{array}$ \\
\hline 18. & $\begin{array}{l}\text { Culm: Attitude (for } \\
\text { floating rice only) }\end{array}$ & NA & 36. & Panicle: Number per plant & Medium (5) \\
\hline
\end{tabular}




\begin{tabular}{|c|c|c|c|c|c|}
\hline 37. & $\begin{array}{l}\text { Spikelet: Colour of tip } \\
\text { of lemma }\end{array}$ & Straw (1) & 50. & $\begin{array}{l}\text { Grain: Weight of } 1000 \\
\text { fully } \\
\text { developed grains }\end{array}$ & $\operatorname{High}(7)$ \\
\hline 38. & $\begin{array}{l}\text { Lemma and Palea: } \\
\text { Colour }\end{array}$ & Absent (1) & 51. & Grain: Length & Short (3) \\
\hline 39. & Panicle: Awns & NA & 52. & Grain: Width & Narrow (3) \\
\hline 40. & $\begin{array}{l}\text { Panicle: Colour of } \\
\text { awns (late } \\
\text { observation) }\end{array}$ & $\mathrm{NA}$ & 53. & $\begin{array}{l}\text { Grain: Phenol reaction of } \\
\text { lemma }\end{array}$ & Absent (1) \\
\hline 41. & $\begin{array}{l}\text { Panicle: Length of } \\
\text { longest awn }\end{array}$ & NA & 54. & Decorticated grain: Length & $\begin{array}{l}\text { Short (5.39 } \\
\mathrm{mm})(1)\end{array}$ \\
\hline 42. & $\begin{array}{l}\text { Panicle: Distribution of } \\
\text { awns }\end{array}$ & Absent (1) & 55. & Decorticated grain: Width & $\begin{array}{l}\text { Medium } \\
(2.19)(5)\end{array}$ \\
\hline 43. & $\begin{array}{l}\text { Panicle : Presence of } \\
\text { secondary } \\
\text { branching }\end{array}$ & NA & 56. & $\begin{array}{l}\text { Decorticated grain: Shape } \\
\text { (in lateral view) }\end{array}$ & Short bold (2) \\
\hline 44. & $\begin{array}{l}\text { Panicle: Secondary } \\
\text { branching }\end{array}$ & Erect (1) & 57. & $\begin{array}{l}\text { Decorticated grain: } \\
\text { Colour }\end{array}$ & $\operatorname{Red}(6)$ \\
\hline 45. & $\begin{array}{l}\text { Panicle: Attitude of } \\
\text { branches }\end{array}$ & $\begin{array}{l}\text { Well exerted } \\
\text { (7) }\end{array}$ & 58. & $\begin{array}{l}\text { Endosperm: Presence of } \\
\text { amylose }\end{array}$ & Present (9) \\
\hline 46. & Panicle: Exertion & Medium (5) & 59. & $\begin{array}{l}\text { Endosperm: Content of } \\
\text { amylose }\end{array}$ & Medium (5) \\
\hline 47. & Time maturity (days) & Medium (5) & 60. & $\begin{array}{l}\text { Varieties with endosperm } \\
\text { of } \\
\text { amylose absent only } \\
\text { Polished grain: Expression } \\
\text { of white core }\end{array}$ & NA \\
\hline 48. & Leaf: Senescence & Straw (1) & 61. & $\begin{array}{l}\text { Gelatinization temperature } \\
\text { through alkali spreading } \\
\text { value }\end{array}$ & $\begin{array}{l}\text { Medium (3) } \\
(4.0)\end{array}$ \\
\hline 49. & Sterile lemma: Colour & Straw (1) & 62. & Decorticated grain: Aroma & Absent (1) \\
\hline
\end{tabular}


Table.5 Salient features of the rice variety Ratnagiri 7 (RTNRR-4)

\begin{tabular}{|c|c|}
\hline Character & Particulars \\
\hline Duration (days) & $\begin{array}{l}\text { 122-125 days (Kharif) } \\
\text { 130-136 (Rabi-hot weather season) }\end{array}$ \\
\hline Plant height $(\mathrm{cm})$ & $100-110$ \\
\hline Lodging & Non-lodging \\
\hline Panicle length $(\mathrm{cm})$ & 22.8 \\
\hline Spikelets / panicle (nos) & $176-190$ \\
\hline Test weight (1000 kernel weight) & $25.8 \mathrm{~g}$ \\
\hline Plant type & Compact \\
\hline Awns & Absent \\
\hline Panicle threshability & Easy \\
\hline Shattering & Non-shattering \\
\hline Scent & Absent \\
\hline Average grain yield (t/ha) & $4.5-5.0$ \\
\hline Potential yields (t/ha) & $8.0-9.0$ \\
\hline Hulling (\%) & 71.10 \\
\hline Milling (\%) & 64.17 \\
\hline Head Rice Recovery (\%) & 60.65 \\
\hline Kernel Length (mm) & 5.39 \\
\hline Kernel Breath (mm) & 2.19 \\
\hline Length and Breath ratio & 2.46 \\
\hline Kernel chalkiness & Very occasionally present \\
\hline Grain type & Short Bold \\
\hline Kernel elongation after cooking (mm) & 9.50 \\
\hline Alkali spreading value & 3.33 \\
\hline Amylose content $(\%)$ & 20.57 \\
\hline Gel consistency $(\mathrm{mm})$ & 89.0 \\
\hline \multicolumn{2}{|l|}{ Reaction to disease and Insect pests } \\
\hline Bacterial leaf blight & Moderately Resistant \\
\hline Leaf blast & Moderately Resistant \\
\hline Neck blast & Moderately resistant \\
\hline Brown plant hopper & Resistant \\
\hline Stem borer & Resistant \\
\hline Leaf folder & Resistant \\
\hline Gall midge & Resistant \\
\hline
\end{tabular}

Table.6 Physical and chemical parameters of RTN RR-4 rice culture analyzed at RARS, Karjat during Kharif 2015 on pooled basis

\begin{tabular}{|c|c|c|c|c|c|c|c|c|}
\hline Entry / IET No. & GT & $\begin{array}{c}\text { Mill } \\
(\%)\end{array}$ & $\begin{array}{c}\text { HRR } \\
(\%)\end{array}$ & $\begin{array}{c}\text { KL } \\
(\mathbf{m m})\end{array}$ & $\begin{array}{c}\text { Grain } \\
\text { Chalk }\end{array}$ & Aroma & $\begin{array}{c}\text { WU } \\
(\mathbf{m l})\end{array}$ & $\begin{array}{c}\text { KLAC } \\
(\mathbf{m m})\end{array}$ \\
\hline RTN RR-4 & SB & 64.17 & 60.65 & 5.71 & OC & NS & 245 & 9.50 \\
\hline Bela $(\mathbf{C h})$ & SB & 63.67 & 56.69 & 5.89 & OC & NS & 175 & 9.00 \\
\hline
\end{tabular}


Continued...

\begin{tabular}{|c|}
\hline Entry / IET No. \\
\hline RTN RR-4 \\
\hline Bela $(\mathrm{Ch})$ \\
\hline
\end{tabular}

\begin{tabular}{|l|l|l|l|l|l}
\hline VER & ER \\
\hline
\end{tabular}

\begin{tabular}{l|l} 
ASV & $\begin{array}{c}\text { AC* } \\
(\%)\end{array}$ \\
\hline
\end{tabular}

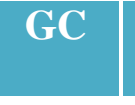

$\mathrm{GT}\left({ }^{1} \mathrm{C}\right)$

\begin{tabular}{|l|l|l|l|l|l|}
\hline 3.78 & 1.66 & 3.33 & 20.57 & 89.0 & High- Intermediate \\
\hline 4.86 & 1.52 & 3.00 & 18.29 & 82.0 & High- Intermediate
\end{tabular}

(Anonymous, 2017)

Hull: Hulling (\%); Mill: Milling (\%); HRR: Head rice recovery (\%); KL: Kernel length (mm); KB: Kernel breadth $(\mathrm{mm})$; L/B: Length and breadth ratio; Grain Chalk: Grain Chalkiness; VOC: Very occasionally present; A: Absent; MS: Medium Slender; ASV: Alkali spreading value; AC: Amylose content (\%); GC: Gel consistency; NS: Non scented

Table.7 Pooled data of iron and zinc analysis at IIRR, Hyderabad

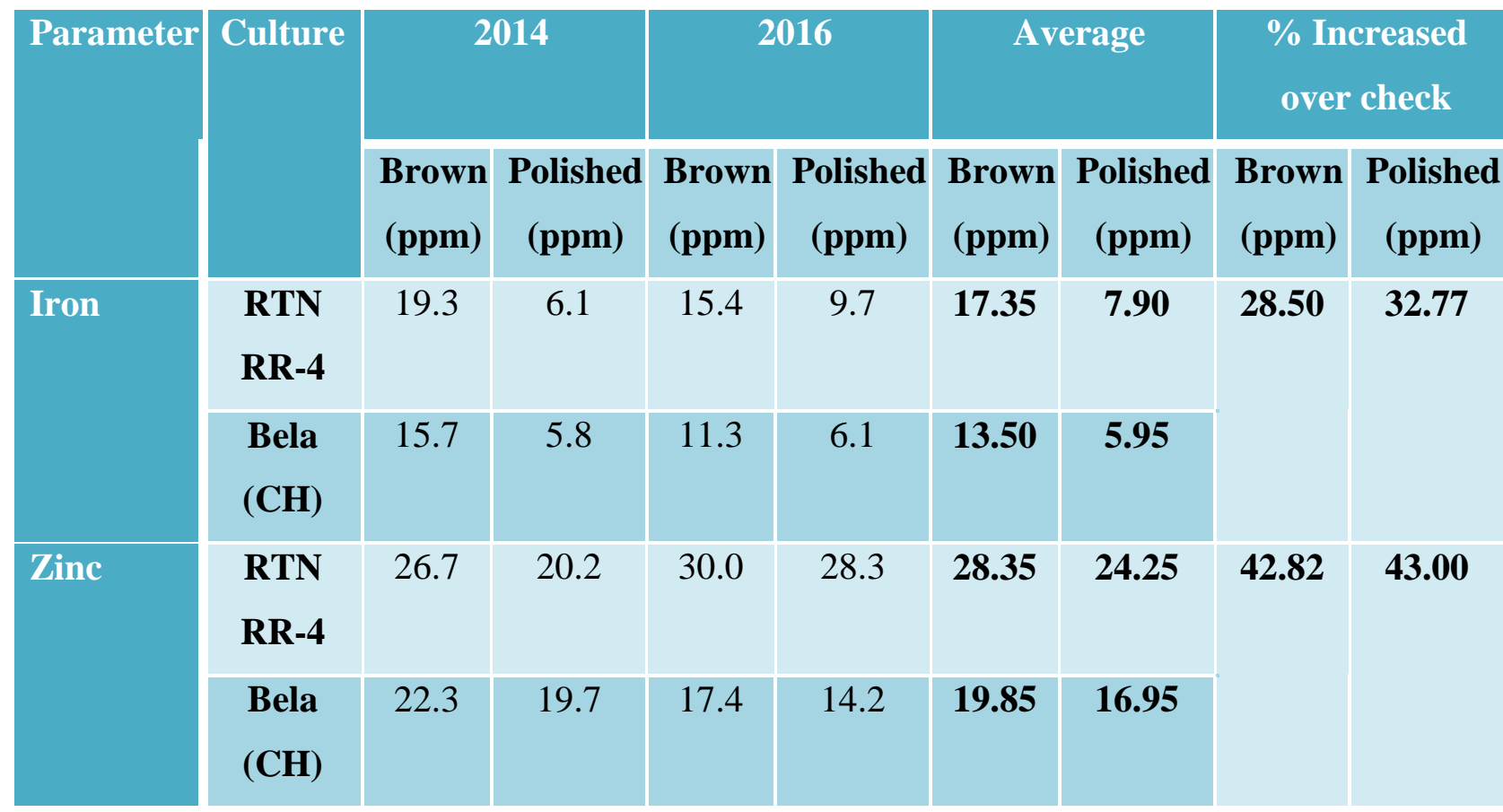

(Anonymous, 2017)

Table.8 Nutritional values of RTN RR-4 at Hi Tech Labs and Consultancy, Sangli (MS)

\begin{tabular}{|c|c|c|c|c|c|c|c|}
\hline $\begin{array}{l}\text { Sr. } \\
\text { No. }\end{array}$ & $\begin{array}{l}\text { Entry } \\
\text { / IET } \\
\text { No. }\end{array}$ & $\begin{array}{c}\text { Total } \\
\text { Carbohydrates } \\
\text { (g per } 100 \mathrm{~g} \\
\text { sample) }\end{array}$ & $\begin{array}{c}\text { Glycemic } \\
\text { Index }\end{array}$ & $\begin{array}{c}\text { Dietary } \\
\text { fibers } \\
\text { (g per } \\
100 \mathrm{~g} \\
\text { sample) }\end{array}$ & $\begin{array}{c}\text { Potassium } \\
\text { (ppm) }\end{array}$ & $\begin{array}{l}\text { Calcium } \\
\text { (ppm) }\end{array}$ & $\begin{array}{l}\text { Riboflavin } \\
\quad \text { (ppm) }\end{array}$ \\
\hline 1 & $\begin{array}{l}\text { RTN } \\
\text { RR-4 }\end{array}$ & 82.29 & 53 & 2.10 & 1120 & 1302 & 0.045 \\
\hline 2 & $\begin{array}{l}\text { Bela } \\
\text { (Ch) }\end{array}$ & 84.11 & 55 & 2.30 & 1834 & 1740 & 0.049 \\
\hline
\end{tabular}

(Anonymous, 2017) 
Table.9 Performance of RTN RR-4 to Bacterial leaf blight and Leaf blast in Maharashtra Screening Nursery-1

(MSN-1) on pooled basis

\begin{tabular}{|c|c|c|c|c|c|c|}
\hline \multirow{2}{*}{$\begin{array}{l}\text { Sr. } \\
\text { No. }\end{array}$} & \multirow{2}{*}{ Name of entry } & \multicolumn{3}{|c|}{ Scale Mean (SI) } & \multirow{2}{*}{$\begin{array}{c}\text { Pooled } \\
\text { Mean (SI) }\end{array}$} & \multirow[t]{2}{*}{ Reaction } \\
\hline & & $K h-2013$ & Kh-2014 & $K h-2015$ & & \\
\hline & Bacterial Leaf blight & (4 Loc) & (3 Loc) & (2 loc) & (9 Loc) & - \\
\hline 1 & RTN RR-4 & 3.5 & 3.33 & 3.00 & 3.33 & MR \\
\hline 2 & Bela $(\mathrm{CH})$ & SNR & 4.67 & 1.00 & 3.20 & MR \\
\hline & Leaf Blast & (5 Loc) & (4 Loc) & (3 Loc) & (12 Loc) & - \\
\hline 1 & RTN RR-4 & 1.8 & 3.00 & 5.33 & 3.08 & MR \\
\hline 2 & Bela $(\mathrm{CH})$ & SNR & 2.75 & 4.67 & 3.57 & MS \\
\hline
\end{tabular}

(Anonymous, 2017)

Table.10 Performance of RTN RR-4 for major insect pests in Maharashtra Screening Nursery-1 (MSN-1) on pooled basis

\begin{tabular}{|c|c|c|c|c|c|c|c|c|c|c|c|c|c|c|c|}
\hline \multirow[t]{4}{*}{$\begin{array}{l}\text { Sr. } \\
\text { No. }\end{array}$} & \multirow[t]{4}{*}{$\begin{array}{c}\text { Name of } \\
\text { entry }\end{array}$} & \multicolumn{4}{|c|}{ Stem Borer (Scale) } & \multirow{2}{*}{\multicolumn{4}{|c|}{ Gall Midge (Scale) }} & \multicolumn{4}{|c|}{ Brown Plant Hopper (Scale) } & \multirow{2}{*}{\multicolumn{2}{|c|}{$\begin{array}{l}\text { Leaf folder } \\
\text { (Scale) } \\
\text { MNC }\end{array}$}} \\
\hline & & \multirow{2}{*}{\multicolumn{2}{|c|}{$\begin{array}{c}\text { Tillering } \\
\text { stage DH } \\
50 \text { DAT \% DH }\end{array}$}} & \multirow{2}{*}{\multicolumn{2}{|c|}{$\begin{array}{c}\text { Panicle } \\
\text { emergence WE } \\
\% \text { WE }\end{array}$}} & & & & & \multirow{2}{*}{\multicolumn{2}{|c|}{$\begin{array}{c}\text { Tillering } \\
\text { Stage } \\
\text { 50 DAT }\end{array}$}} & \multirow{2}{*}{\multicolumn{2}{|c|}{$\begin{array}{c}\text { Panicle } \\
\text { emergence } \\
\text { 70 DAT }\end{array}$}} & & \\
\hline & & & & & & & DAT & & DAT & & & & & & \\
\hline & & Mean & Reaction & Mean & Reaction & Mean & Reaction & Mean & Reaction & Scale & Reaction & Scale & Reaction & $\% \mathrm{DL}$ & scale \\
\hline 1 & $\begin{array}{l}\text { RTN } \\
\text { RR-4 }\end{array}$ & 0.3 & HR & 2.0 & $\mathrm{R}$ & 2.3 & $\mathrm{R}$ & 4.3 & MS & 1 & $\mathrm{R}$ & 1 & $\mathrm{R}$ & 6.6 & MR \\
\hline 2 & $\begin{array}{l}\text { Bela } \\
(\mathrm{CH})\end{array}$ & 1.0 & HR & 5.0 & MS & 1.5 & HR & 3.0 & MR & 1 & $\mathrm{R}$ & 1 & $\mathrm{R}$ & 19.9 & MR \\
\hline
\end{tabular}

\begin{tabular}{|c|c|l|c|c|l|l|} 
Scale & Code & Reaction & & Scale & Code & Reaction \\
\hline $\mathbf{0}$ & HR & Highly Resistant & & $4-6$ & MS & Moderately Susceptible \\
\hline $\mathbf{1}$ & HR & Highly Resistant & & 7 & S & Susceptible \\
\hline 2 & R & Resistant & & $8-9$ & HS & Highly Susceptible \\
\hline 3 & MR & Moderately Resistant & & & & \\
\hline
\end{tabular}


The variety Ratnagiri 7 showed higher milling $(64.17 \%)$ and head rice recovery $(60.65 \%)$. The kernel length $(5.39 \mathrm{~mm})$, kernel breadth $(2.19 \mathrm{~mm})$, length : breadth ratio $(2.46)$ and translucent kernel observed to be an inherited traits in this rice variety which contribute to higher milling and head rice recovery in Ratnagiri 7 (Red rice) rice variety (Table 6) (Bhattacharya, 1980).

Ratnagiri 7 (Red rice) rice variety recorded an intermediate amylose content (20.57 \%) indicating better cooking qualities of kernels (Anonymous, 2017).

The variety showed medium gel consistency $(23 \mathrm{~mm})$ and Alkali spreading value was 3.33. Amylose content between 20-25\% is intermediate and good for cooking, 61-100 $\mathrm{mm}$ gel consistency is soft and alkali spreading value ranged 3-5 is high to intermediate good for cooking (Shobha Rani, 2003).

The above observation indicates that the new variety Ratnagiri 7 meets the requirements of millers and consumers for higher monitory returns to farmers.

The rice variety Ratnagiri 7 (Red rice) was screened for reaction to various diseases and insect pests at endemic locations in the state. The variety showed moderately resistant to Moderately resistant to leaf blast, bacterial leaf blight (Table 8).

While, it was also recorded resistance reaction to neck stem borer, leaf folder and gall midge under endemic test locations (Table 9) (Anonymous, 2017).

In view of higher yields, superior grain quality and field tolerance to major insect pests and diseases, the rice variety Ratnagiri 7 (Red rice) (IET-25448) recommended to release for commercial cultivation in Konkan region of Maharashtra during the year 2017. It will meet the requirement of farmers and consumers in the state.

\section{Acknowledgements}

The authors are thankful to the scientists and technical staff who helped in screening and evaluation in various trials at different locations in the state and country.

\section{References}

Anonymous, 2017. Release proposal of Ratnagiri -7, presented during Joint Agresco, 2017 held on 28-31 $1^{\text {st }}$ May, 2017 at V.N.M. A.U., Parbhani. pp: 132.

Arumugasamy, S., Jayashankar, N., Subramanian, K., Sridhar, S., and Vijayalakshmi, K. 2001. Indigenous Rice Varieties. Centre for Indian Knowledge Systems (CIKS), Chennai, Tamil Nadu, India. pp. 74-88.

Bhattacharya, K. R. (1980). Breakage of rice during milling: A review. Tropical science, Vol. 22: 255-276.

Chaudhary, R.C. and Tran, D.V. 2001. Specialty rices of the world - a prologue. In: Specialty Rices of the World: Breeding, Production, and Marketing Chaudhary, R.C. and Tran, D.V., eds.). FAO, Rome, Italy; and Oxford IBH Publishers, India. pp. 3-14.

Panse, V. G. and P. V. Sukhatme. (1967). Statistical methods for Agricultural workers. ICAR. Publ. New Delhi (India).

Rani, S. and Krishnaiah, K. 2001. Current status and future prospects of improving traditional aromatic rices. In: Specialty Rices of the World: Breeding, Production, and Marketing (Chaudhary, R.C. and Tran, D.V., eds.). FAO, Rome, Italy; and Oxford IBH Publishers, India. pp. 49-79. 
Shobha Rani, N. (2003). Quality considerations in developing rice hybrids. Proceed Winter school on "Advances in hybrid rice technology" held at DRR, Hyderabad during the Sept., 10-30, 2003: 145-157.

\section{How to cite this article:}

Waghmode, B.D., N.G. Sonone, V.C. Navhale, S.R. Kadam and Bhave, S.G. 2018. Ratnagiri 7 - Red Kernel, Semi Dwarf and High Yielding Rice Variety for Konkan Region of Maharashtra State in India. Int.J.Curr.Microbiol.App.Sci. 7(04): 3442-3453.

doi: https://doi.org/10.20546/ijcmas.2018.704.389 